\title{
A Note on Euclidean Ramsey Theory
}

\author{
James H. Schmerl \\ Department of Mathematics, University of Connecticut, \\ Storrs, CT 06269-3009, USA \\ schmerl@math.uconn.edu
}

\begin{abstract}
Suppose $n \geq 2$ and $X, Y \subseteq \mathbb{R}^{n}$ are such that $X$ is infinite and $Y$ is the set of vertices of an $n$-simplex. Then there is blue/red coloring of $\mathbb{R}^{n}$ such that no set similar to $X$ is monochromatically blue and no set similar to $Y$ is monochromatically red.
\end{abstract}

Consider some finite-dimensional Euclidean space $\mathbb{R}^{n}$. If $X_{0}, X_{1}, \ldots, X_{k-1} \subseteq \mathbb{R}^{n}$, then we write

$$
\mathbb{R}^{n} \longrightarrow\left(X_{0}, X_{1}, \ldots, X_{k-1}\right)
$$

if for any function $\psi: \mathbb{R}^{n} \longrightarrow k$ there are $i<k$ and $H \subseteq \mathbb{R}^{n}$ such that $H$ is similar to $X_{i}$ and $\psi$ is constantly $i$ on $H$. If $\mathbb{R}^{n} \longrightarrow\left(X_{0}, X_{1}, \ldots, X_{k-1}\right)$ is false, then we write $\mathbb{R}^{n} \nrightarrow\left(X_{0}, X_{1}, \ldots, X_{k-1}\right)$.

The following theorem, but with the stronger conclusion with "homothetic" replacing "similar", is the Gallai-Witt Theorem, proved independently by Gallai (see [6] ${ }^{1}$ ) and Witt [9].

Theorem A. If $X_{0}, X_{1}, \ldots, X_{k-1} \subseteq \mathbb{R}^{n}$ are finite, then $\mathbb{R}^{n} \longrightarrow\left(X_{0}, X_{1}, \ldots, X_{k-1}\right)$.

For infinite sets, Theorem A fails as shown by the following easy consequence of the much stronger Theorem 19 of [4].

Theorem B. If $X \subseteq \mathbb{R}^{n}$ is infinite, then $\mathbb{R}^{n} \nrightarrow(X, X)$.

\footnotetext{
${ }^{1}$ The result of Gallai (Grünwald) as quoted on page 123 of [6] is restricted to lattice points. However, on page 130 of [6] there is stated and proved a theorem that is slightly stronger than even the homothetic version of Theorem A.
} 
Theorem B shows that the symmetric 2-color version of Theorem A fails for an infinite set. Similar arguments can be used to show the failure of the asymmetric version; specifically, if $X, Y \subseteq \mathbb{R}^{n}$ are infinite, then $\mathbb{R}^{n} \nrightarrow(X, Y)$. Baumgartner [1] proved the following theorem showing the asymmetric version can fail even if only one of the sets is infinite.

Theorem C. If $X, Y \subseteq \mathbb{R}^{n}$ are such that $X$ is an infinite arithmetic progression and $Y$ is a three-element arithmetic progression, then $\mathbb{R}^{n} \nrightarrow(X, Y)$.

An affine subspace of $\mathbb{R}^{n}$ is a translate of a subspace of $\mathbb{R}^{n}$. For example, a line is just a one-dimensional affine subspace. If $Y \subseteq \mathbb{R}^{n}$, then let aff $(Y)$ be the affine subspace generated by $Y$. In general, $|Y| \geq \operatorname{dim}(\operatorname{aff}(Y))+1$, but if $|Y|=\operatorname{dim}(\operatorname{aff}(Y))+1$, then $Y$ is (affinely) independent, and if $|Y|>\operatorname{dim}(\operatorname{aff}(Y))+1$, then $Y$ is dependent. For example, every three-element arithmetic progression is dependent. If $Y \subseteq \mathbb{R}^{n}$ and $k<\omega$, then $Y$ is (the set of vertices of) a $k$-simplex iff $|Y|=k+1$ and $Y$ is independent.

The following theorem is the main new result.

Theorem D. If $n \geq 2$ and $X, Y \subseteq \mathbb{R}^{n}$ are such that $X$ is infinite and $Y$ is an $n$-simplex, then $\mathbb{R}^{n} \nrightarrow(X, Y)$.

Theorem D answers a question of Komjáth [5], who proved the special case in which $n=2$. His proof was along the lines of Ceder's proof in [2] and made use of Theorem C. As pointed out by Komjáth [5], Theorem D is, in some sense, an optimal result as shown by the following proposition which is easily proved by induction.

Proposition E. If $X, Y \subseteq \mathbb{R}^{n}$ are such that $X$ is a circle, $Y$ is independent, and $|Y| \leq n$, then $\mathbb{R}^{n} \longrightarrow(X, Y)$.

It would be interesting to have a characterization of those countable subsets $X, Y \subseteq \mathbb{R}^{n}$ for which $\mathbb{R}^{n} \longrightarrow(X, Y)$.

\section{Preliminaries}

We use $\omega$ to denote the set of natural numbers, and we systematically write $n<\omega$ instead of $n \in \omega$. As usual, each natural number is the set of smaller natural numbers; that is, $n=\{0,1, \ldots, n-1\}$. Throughout this paper we consider a fixed $n$-dimensional Euclidean space $\mathbb{R}^{n}$, where $2 \leq n<\omega$. For $x \in \mathbb{R}^{n}$, we let $\|x\|$ be the usual Euclidean norm of $x$. If $X \subseteq \mathbb{R}$, then we write $X=\left\{x_{0}, x_{1}, x_{2}, \ldots, x_{m-1}\right\}_{<}$to indicate that $x_{0}<x_{1}<x_{2}<\cdots<x_{m-1}$. We use the notational convention that if we consider a point in $\mathbb{R}^{m}$, say $t \in \mathbb{R}^{m}$, then we let $t=\left(t_{0}, t_{1}, \ldots, t_{m-1}\right)$.

We always have in mind, for this paper, a fixed countable, real-closed subfield $\mathbb{F} \subseteq \mathbb{R}$ and also a transcendence basis $\mathcal{T}$ for $\mathbb{R}$ over $\mathbb{F}$. (A transcendence basis can easily be shown to exist since we are assuming the Axiom of Choice.) If $D \subseteq \mathbb{R}$, then we say that the $k$-ary relation $R \subseteq \mathbb{R}^{k}$ is $D$-definable if it is definable by a first-order formula in the ordered field $(\mathbb{R},+, \times, 0,1,<)$ using parameters just from $D \cup \mathbb{F}$. If $R$ is $\emptyset$ - 
definable, then we say simply that it is definable. If $B \subseteq \mathbb{R}^{m}$ and we write something like $f: B \longrightarrow \mathbb{R}$, then we understand that $f$ is a function whose domain $\operatorname{dom}(f)=B$. Therefore, if $f$ is $D$-definable, then its domain $B$ must also be $D$-definable. Notice that $R$ is semi-algebraic iff it is $\mathbb{R}$-definable.

Definable analytic functions are crucial to our method. The properties that we need about these functions are basic properties that follow from the o-minimality of $\mathbb{R}$, which is a consequence of the Tarski-Seidenberg Theorem on the elimination of quantifiers. The book by van den Dries [3] is a suggested reference.

The following basic facts about definable analytic functions are crucial for our method. Proofs of the following results can be found in [8].

Proposition 1.1. Supppose $f: U \longrightarrow \mathbb{R}$ is a definable analytic function, where $U \subseteq$ $\mathbb{R}^{m}$ is an open connected set. Let $t_{0}, t_{1}, t_{2}, \ldots, t_{m-1} \in \mathcal{T}$ be distinct such that $t \in U$ and $f(t)=0$. Then $f$ is identically 0 on $U$.

Corollary 1.2. Supppose $f: U \longrightarrow \mathbb{R}$ is a definable analytic function, where $U \subseteq \mathbb{R}^{m}$ is an open convex set. Let $t_{0}, t_{1}, t_{2}, \ldots, t_{m-1} \in \mathcal{T}$ (not necessarily distinct) be such that $t \in U$ and $f(t)=0$. Let $u \in U$ be such that whenever $i<j<m$ and $t_{i}=t_{j}$, then $u_{i}=u_{j}$. Then $f(u)=0$.

Proposition 1.3. Supppose $f: U \longrightarrow \mathbb{R}$ is a definable function, where $U \subseteq \mathbb{R}^{m}$ is open, and $t_{0}, t_{1}, t_{2}, \ldots, t_{m-1} \in \mathcal{T}$ are distinct such that $t \in U$. Then there is an open neighborhood $V$ of $t$ such that $f \uparrow V$ is an analytic function.

If $X \subseteq \mathbb{R}^{n}$, then a function $\alpha: X \longrightarrow \mathbb{R}^{n}$ is a similarity if there is a positive $c \in \mathbb{R}$ such that whenever $x, y \in X$, then $\|\alpha(x)-\alpha(y)\|=c\|x-y\|$. The number $c$ (which is unique as long as $|X| \geq 2$ ) is the scaling factor of $\alpha$.

A function on $\mathbb{R}^{n}$ will sometimes be referred to as a coloring with the concomitant terminology. For example, subsets of $\mathbb{R}^{n}$ on which the coloring is constant are referred to as monochromatic. With this terminology, $\mathbb{R}^{n} \longrightarrow(X, Y)$ means that whenever $\psi$ is a 2-coloring of $\mathbb{R}^{n}$, then either there is a subset $X^{\prime} \subseteq \mathbb{R}^{n}$ similar to $X$ on which $\chi$ is monochromatically 0 or there is a subset $Y^{\prime} \subseteq \mathbb{R}^{n}$ similar to $Y$ on which $\psi$ is monochromatically 1. A 2-coloring $\psi$ confirms that $\mathbb{R}^{n} \nrightarrow(X, Y)$ if, whenever $X^{\prime}, Y^{\prime} \subseteq$ $\mathbb{R}^{n}$ are similar to $X, Y$ respectively, then $\psi$ is neither monochromatically 0 on $X^{\prime}$ nor monochromatically 1 on $Y^{\prime}$.

\section{Acceptable Colorings}

In this section we continue the assumptions of the previous section that $2 \leq n<\omega$, $\mathbb{F} \subseteq \mathbb{R}$ is a countable, real-closed field, and $\mathcal{T} \subseteq \mathbb{R}$ is a transcendence basis for $\mathbb{R}$ over $\mathbb{F}$.

It is known [7] that there is a countable coloring of $\mathbb{R}^{n}$ for which there are no monochromatic equilateral triangles. (For $n=2$, this had been previously proved by Ceder [2].) The proof is easily adapted to a proof of the stronger result that for any given countable set of triangles, there is a countable coloring of $\mathbb{R}^{n}$ that has no monochromatic trian- 
gle similar to any in the countable set. The result in [7] was subsequently improved in [8] to isosceles triangles: there is a countable coloring of $\mathbb{R}^{n}$ for which there are no monochromatic isosceles triangles. A somewhat stronger result is Lemma 2.1.

If $R \subseteq \mathbb{R}^{k}$ is $\mathbb{R}$-definable, then there is a unique, minimal subset $T \subseteq \mathcal{T}$ such that $R$ is $T$-definable; we call this set $T$ the support of $R$ and denote it by $\operatorname{supp}(R)$. If $a \in \mathbb{R}^{k}$, then we usually write $\operatorname{supp}(a)$ instead of $\operatorname{supp}(\{a\})$. Notice that if $a=\left(a_{0}, a_{1}, \ldots, a_{k-1}\right) \in$ $\mathbb{R}^{k}$, then $\operatorname{supp}(a)=\operatorname{supp}\left(a_{0}\right) \cup \operatorname{supp}\left(a_{1}\right) \cup \cdots \cup \operatorname{supp}\left(a_{k-1}\right)$.

If $a \in \mathbb{R}^{k}$ and $\operatorname{supp}(a)=\left\{t_{0}, t_{1}, \ldots, t_{m-1}\right\}_{<}$, then there is a definable open neighborhood $U \subseteq \mathbb{R}^{m}$ of $\left(t_{0}, t_{1}, \ldots, t_{m-1}\right)$ and a definable analytic function $f: U \longrightarrow \mathbb{R}^{k}$ such that $f\left(t_{0}, t_{1}, \ldots, t_{m-1}\right)=a$.

We wish to place a restriction on the domains of the analytic functions. If $I, J \subseteq \mathbb{R}$ are intervals, then we write $I<J$ if $x<y$ whenever $x \in I$ and $y \in J$. If $k<\omega$, then we say that the interval $I \subseteq \mathbb{R}$ is a dyadic interval with norm $k$ if there is an integer $i$ such that $I$ is the bounded, open interval $\left(i / 2^{k},(i+1) / 2^{k}\right)$. If $m<\omega$ and $B \subseteq \mathbb{R}^{m}$, then $B$ is an $m$-box with norm $k$ if there are dyadic intervals $I_{0}<I_{1}<\cdots<I_{m-1}$, each having norm $k$, such that $B=I_{0} \times I_{1} \times \cdots \times I_{m-1}$. If $B$ is an $m$-box for some $m<\omega$, then $B$ is a box. We let $\mathcal{C}$ be the set of all definable analytic functions $f: B \longrightarrow \mathbb{R}^{n}$, where $B$ is a box. Clearly, $\mathcal{C}$ is countable. The norm of a function in $\mathcal{C}$ is the norm of its domain.

There is a small technical point that needs clarification. If $m=0$, then $B=\{\emptyset\}$ is the unique $m$-box. For our purposes, the only definable analytic functions $f: B \longrightarrow \mathbb{R}^{n}$, where $B$ is the 0-box, are those for which $f: B \longrightarrow \mathbb{F}^{n}$. Since the norm of this box $B$ is not well defined, we conventionally let $v(B)=-\infty$.

If $X$ is either a dyadic interval, a box, or a function in $\mathcal{C}$, then we let $v(X)$ be its norm.

If $a \in \mathbb{R}^{n}$, then $f$ is an acceptable color for $a$ if $f \in \mathcal{C}, \operatorname{supp}(a)=\left\{t_{0}, t_{1}, \ldots, t_{m-1}\right\}_{<}$, $\left(t_{0}, t_{1}, \ldots, t_{m-1}\right) \in \operatorname{dom}(f)$, and $f\left(t_{0}, t_{1}, \ldots, t_{m-1}\right)=a$. A function $\chi: \mathbb{R}^{n} \longrightarrow \mathcal{C}$ is an acceptable coloring if, whenever $a \in \mathbb{R}^{n}$, then $\chi(a)$ is an acceptable color for $a$.

For each $a \in \mathbb{R}^{n}$, it is always possible to find an acceptable color for $a$. Moreover, there are infinitely many acceptable colors for $a$. For, if $f \in \mathcal{C}$ is an acceptable color for $a$, where $v(f)=k$, then for each $\ell \geq k$, there is an acceptable color $g \in \mathcal{C}$ such that $v(g)=\ell$. (In fact, for each $\ell \geq k$ there is exactly one $g$ that is acceptable for $a$ such that $\nu(g)=\ell$, and it must be that $g \subseteq f$.) Therefore, there are many acceptable colorings.

Lemma 2.1. Let $\chi: \mathbb{R}^{n} \longrightarrow \mathcal{C}$ be an acceptable coloring. Suppose $a, b, c \in \mathbb{R}^{n}$ are distinct points and $r \in \mathbb{F}$ is such that $\|a-b\|=r\|b-c\|$. Then $\chi(a) \neq \chi(b)$ or $\chi(b) \neq \chi(c)$.

Proof. Suppose $\chi(a)=\chi(b)=\chi(c)=f$, where $\operatorname{dom}(f)=B \subseteq \mathbb{R}^{m}$. Let $F: B^{3} \longrightarrow \mathbb{R}$ be the definable analytic function

$$
F(x, y, z)=\|f(x)-f(y)\|^{2}-r^{2}\|f(y)-f(z)\|^{2} .
$$

Thus, $F(x, y, z)=0$ iff $\|f(x)-f(y)\|=r\|f(y)-f(z)\|$. Let

$$
\begin{aligned}
& \operatorname{supp}(a)=\left\{t_{0}, t_{1}, \ldots, t_{m-1}\right\}_{<}, \\
& \operatorname{supp}(b)=\left\{u_{0}, u_{1}, \ldots, u_{m-1}\right\}_{<}, \\
& \operatorname{supp}(c)=\left\{v_{0}, v_{1}, \ldots, v_{m-1}\right\}_{<} .
\end{aligned}
$$


Hence, $f(t)=a, f(u)=b$, and $f(v)=c$. Then $F(t, u, v)=0$. Since $a \neq c$, there is $j<m$ such that $t_{j} \neq v_{j}$ and, therefore, $t_{j} \neq u_{j}$ or $u_{j} \neq v_{j}$. Without loss of generality, we assume that $t_{j} \neq u_{j}$. Let $w \in \mathbb{R}^{m}$ agree with $t$ except that $w_{j}=u_{j}$. (This means $w_{i}=t_{i}$ if $j \neq i<m$ and $w_{i}=u_{i}$ if $i=j$.) Then $w \in B$, so it follows from Corollary 1.2 that $F(t, w, w)=0$. Thus, $\|a-f(w)\|=r\|f(w)-f(w)\|=0$. Therefore, $a=f(w)$ and $t_{j} \notin\left\{w_{0}, w_{1}, \ldots, w_{m-1}\right\} \subseteq \mathcal{T}$, contradicting that $t_{j} \in \operatorname{supp}(a)$.

We say that a subset $\mathcal{C}_{0} \subseteq \mathcal{C}$ is thin if, for each $\ell<\omega,\left\{f \in \mathcal{C}_{0}: v(f)=\ell\right\}$ is finite.

Lemma 2.2. Let $\chi: \mathbb{R}^{n} \longrightarrow \mathcal{C}$ be an acceptable coloring. Let $f_{0}, f_{1}, \ldots, f_{k-1} \in \mathcal{C}$, and let $F:\left(\mathbb{R}^{n}\right)^{k} \longrightarrow \mathbb{R}^{n}$ be a definable analytic function. Then there is a thin $\mathcal{C}_{0} \subseteq \mathcal{C}$ such that whenever $a_{0}, a_{1}, \ldots, a_{k-1} \in \mathbb{R}^{n}$ are such that $\chi\left(a_{i}\right)=f_{i}$ for each $i<k$, then $\chi\left(F\left(a_{0}, a_{1}, \ldots, a_{k-1}\right)\right) \in \mathcal{C}_{0}$.

Proof. For each $i<k$, let $\operatorname{dom}\left(f_{i}\right)=B_{i}=I_{i 0} \times I_{i 1} \times \cdots \times I_{i m_{i}-1} \subseteq \mathbb{R}^{m_{i}}$. Let $m=m_{0}+m_{1}+\cdots+m_{k-1}$, and then let $B=B_{0} \times B_{1} \times \cdots \times B_{k-1} \subseteq \mathbb{R}^{m}$. Let $G: B \longrightarrow \mathbb{R}^{n}$ be the definable analytic function such that

$$
G\left(x_{0}, x_{1}, \ldots, x_{k-1}\right)=F\left(f_{0}\left(x_{0}\right), f_{1}\left(x_{1}\right), \ldots, f_{k-1}\left(x_{k-1}\right)\right)
$$

whenever $\left(x_{0}, x_{1}, \ldots, x_{k-1}\right) \in B$.

Let $\mathcal{G}$ be the smallest set of functions $H: J_{0} \times J_{1} \times \cdots \times J_{\ell-1} \longrightarrow \mathbb{R}$, with each $J_{i}$ being a dyadic interval, such that $G \in \mathcal{G}$ and $\mathcal{G}$ has the closure properties that $H^{\prime} \in \mathcal{G}$ whenever $H \in \mathcal{G}$ and one of the following holds:

(1) $H^{\prime}$ is obtained from $H$ by permuting the coordinates in the domain.

(2) $\operatorname{dom}(H)=J_{0} \times J_{1} \times \cdots \times J_{\ell}, J^{\prime}$ is one of the halves of $J_{\ell}, \operatorname{dom}\left(H^{\prime}\right)=$ $J_{0} \times J_{1} \times \cdots \times J_{\ell-1} \times J^{\prime}$, and $H^{\prime} \subseteq H$.

(3) $\operatorname{dom}(H)=J_{0} \times J_{1} \times \cdots \times J_{\ell}, \operatorname{dom}\left(H^{\prime}\right)=J_{0} \times J_{1} \times \cdots \times J_{\ell-1}, q$ is the midpoint of $J_{\ell}$, and

$$
H^{\prime}\left(x_{0}, x_{1}, \ldots, x_{\ell-1}\right)=H\left(x_{0}, x_{1}, \ldots, x_{\ell-1}, q\right) .
$$

(4) $\operatorname{dom}(H)=J_{0} \times J_{1} \times \cdots \times J_{\ell}, \operatorname{dom}\left(H^{\prime}\right)=J_{0} \times J_{1} \times \cdots \times J_{\ell-1}, J_{\ell}=J_{\ell-1}$ and $H^{\prime}\left(x_{0}, x_{1}, \ldots, x_{\ell-1}\right)=H\left(x_{0}, x_{1}, \ldots, x_{\ell-2}, x_{\ell-1}, x_{\ell-1}\right)$.

Let $\mathcal{C}_{0}=\mathcal{G} \cap \mathcal{C}$. It is easily checked that $\mathcal{C}_{0}$ is thin.

It can be verified that whenever $a_{0}, a_{1}, \ldots, a_{k-1} \in \mathbb{R}^{n}$ are such that $\chi\left(a_{i}\right)=f_{i}$ for each $i<k$, then $\chi\left(F\left(a_{0}, a_{1}, \ldots, a_{k-1}\right)\right) \in \mathcal{C}_{0}$. Modulo some details, this completes the proof.

Two special cases of this lemma are worth noting, each requiring a lemma. (The second of these special cases will be used in the proof of Theorem D in the last section. Although the first will not be used in the proof of Theorem D, its lemma will be.)

Lemma 2.3. Let $Y=\left\{y_{0}, y_{1}, \ldots, y_{n}\right\} \subseteq \mathbb{F}^{n}$ be an $n$-simplex, and let $a \in \mathbb{F}^{n}$. Then there is a definable analytic function $F:\left(\mathbb{R}^{n}\right)^{n+1} \longrightarrow \mathbb{R}^{n}$ such that whenever $\alpha: \mathbb{R}^{n} \longrightarrow$ 
$\mathbb{R}^{n}$ is a similarity, then

$$
\alpha(a)=F\left(\alpha\left(y_{0}\right), \alpha\left(y_{1}\right), \ldots, \alpha\left(y_{n}\right)\right) .
$$

Proof. There are uniquely determined $r_{0}, r_{1}, \ldots, r_{n} \in \mathbb{R}$ such that $r_{0}+r_{1}+\cdots+r_{n}=1$ and $a=r_{0} y_{0}+r_{1} y_{1}+\cdots+r_{n} y_{n}$. Let $F:\left(\mathbb{R}^{n}\right)^{n+1} \longrightarrow \mathbb{R}^{n}$ be such that whenever $x_{0}, x_{1}, \ldots, x_{n} \in \mathbb{R}^{n}$, then

$$
F\left(x_{0}, x_{1}, \ldots, x_{n}\right)=r_{0} x_{0}+r_{1} x_{1}+\cdots+r_{n} x_{n} .
$$

Then $F$ is definable since each $r_{i} \in \mathbb{F}$. Clearly, $F$ is as required.

Corollary 2.4. Let $\chi: \mathbb{R}^{n} \longrightarrow \mathcal{C}$ be an acceptable coloring. Let $\left\{y_{0}, y_{1}, \ldots, y_{n}\right\} \subseteq \mathbb{F}^{n}$ be an $n$-simplex, let $a \in \mathbb{F}^{n}$, and let $f_{0}, f_{1}, \ldots, f_{n} \in \mathcal{C}$. Then there is a thin $\mathcal{C}_{0} \subseteq \mathcal{C}$ such that whenever $\alpha: \mathbb{R}^{n} \longrightarrow \mathbb{R}^{n}$ is a similarity such that $\chi\left(\alpha\left(y_{i}\right)\right)=f_{i}$ for $i \leq n$, then $\chi(\alpha(a)) \in \mathcal{C}_{0}$.

Lemma 2.5. Let $\left\{y_{0}, y_{1}, \ldots, y_{n}\right\} \subseteq \mathbb{F}^{n}$ be an $n$-simplex. Then there are definable analytic functions $G, G^{\prime}:\left(\mathbb{R}^{n}\right)^{n} \longrightarrow \mathbb{R}^{n}$ such that whenever $\alpha: \mathbb{R}^{n} \longrightarrow \mathbb{R}^{n}$ is a similarity, then

$$
\alpha\left(y_{n}\right)=G\left(\alpha\left(y_{0}\right), \alpha\left(y_{1}\right), \ldots, \alpha\left(y_{n-1}\right)\right)
$$

or

$$
\alpha\left(y_{n}\right)=G^{\prime}\left(\alpha\left(y_{0}\right), \alpha\left(y_{1}\right) \ldots, \alpha\left(y_{n-1}\right)\right) .
$$

Proof. Let $N$ : $\left(\mathbb{R}^{n}\right)^{n-1} \longrightarrow \mathbb{R}^{n}$ be the $n$-dimensional analogue of the cross-product. ${ }^{2}$ Then $N$ is a definable analytic function such that if $z_{1}, z_{2}, \ldots, z_{n-1} \in \mathbb{R}^{n}$ are linearly independent vectors, then $N\left(z_{1}, z_{2}, \ldots, z_{n-1}\right)$ is a nonzero vector that is orthogonal to each of $z_{1}, z_{2}, \ldots, z_{n-1}$, and if $z_{1}, z_{2}, \ldots, z_{n-1}$ are linearly dependent, then $N\left(z_{1}\right.$, $\left.z_{2}, \ldots, z_{n-1}\right)$ is the zero vector. Let $r_{1}, r_{2}, \ldots, r_{n} \in \mathbb{R}$ be such that $y_{n}-y_{0}=r_{1}\left(y_{1}-\right.$ $\left.y_{0}\right)+r_{2}\left(y_{2}-y_{0}\right)+\cdots+r_{n-1}\left(y_{n-1}-y_{0}\right)+r_{n} N\left(y_{1}-y_{0}, y_{2}-y_{0}, \ldots, y_{n-1}-y_{0}\right)$. Clearly, each $r_{i} \in \mathbb{F}$. Now we define $G, G^{\prime}$ so that

$$
G\left(x_{0}, x_{1}, \ldots, x_{n-1}\right)=r_{1} z_{1}+r_{2} z_{2}+\cdots+r_{n-1} z_{n-1}+r_{n} N\left(z_{1}, z_{2}, \ldots, z_{n-1}\right)+x_{0},
$$

and

$$
G^{\prime}\left(x_{0}, x_{1}, \ldots, x_{n-1}\right)=r_{1} z_{1}+r_{2} z_{2}+\cdots+r_{n-1} z_{n-1}-r_{n} N\left(z_{1}, z_{2}, \ldots, z_{n-1}\right)+x_{0},
$$

where $z_{i}=x_{i}-x_{0}$.

It can be checked that $G, G^{\prime}$ are as required.

\footnotetext{
${ }^{2}$ To be definitive, suppose $z_{1}, z_{2}, \ldots, z_{n-1} \in \mathbb{R}^{n}$. Let $M$ be the $(n-1) \times n$ matrix whose $i$ th row is $z_{i}$. For $1 \leq j \leq n$, let $M_{j}$ be the $(n-1) \times(n-1)$ submatrix of $M$ obtained by deleting its $j$ th column. Then let $N\left(z_{1}, z_{2}, \ldots, z_{n-1}\right)$ be the vector in $\mathbb{R}^{n}$ whose $j$ th coordinate is $(-1)^{j+1} \operatorname{det}\left(M_{j}\right)$.
} 
Corollary 2.6. Let $\chi: \mathbb{R}^{n} \longrightarrow \mathcal{C}$ be an acceptable coloring. Let $\left\{y_{0}, y_{1}, \ldots, y_{n}\right\} \subseteq \mathbb{F}^{n}$ be an n-simplex, and let $f_{0}, f_{1}, \ldots, f_{n-1} \in \mathcal{C}$. Then there is a thin $\mathcal{C}_{1} \subseteq \mathcal{C}$ such that whenever $\alpha: \mathbb{R}^{n} \longrightarrow \mathbb{R}^{n}$ is a similarity such that $\chi\left(\alpha\left(y_{i}\right)\right)=f_{i}$ for $i<n$, then $\chi\left(\alpha\left(y_{n}\right)\right) \in \mathcal{C}_{1}$.

\section{Preferred Colorings}

In this section we continue with the assumptions of the previous sections that $\mathbb{F}$ is a countable real-closed subfield of $\mathbb{R}$ and $\mathcal{T}$ is a transcendence basis for $\mathbb{R}$ over $\mathbb{F}$. Some definitions are needed. A subset $S \subseteq \mathbb{R}^{n}$ is an $(n-1)$-sphere with radius $r$ and center $c$ if $0<r \in \mathbb{R}, c \in \mathbb{R}^{n}$ and $S=\left\{x \in \mathbb{R}^{n}:\|x-c\|=r\right\}$. More generally, if $k<n$, then $S$ is a $k$-sphere with radius $r$ and center $c$ if $c \in \operatorname{aff}(S)$ and there is an $(n-1)$-sphere $S^{\prime}$ with radius $r$ and center $c$ such that $S=S^{\prime} \cap \operatorname{aff}(S)$ and $\operatorname{dim}(\operatorname{aff}(S))=k+1$. By convention, we let $\mathbb{R}^{n}$ be the (unique) $n$-sphere; we arbitrarily let the origin be its center and 1 its radius.

If $S$ is a $k$-sphere for some $k \leq n$, then $S$ is a sphere. A circle is a 1 -sphere. If $S$ is a sphere, then its axis is $\left\{a \in \mathbb{R}^{n}: \forall x, y \in S,\|x-a\|=\|y-a\|\right\}$. If $k<n$ and $S$ is a sphere, then $S$ is a $k$-sphere iff its axis is an $(n-k-1)$-dimensional affine subspace.

Now consider some $a \in \mathbb{R}^{n}$. Let $\operatorname{supp}(a)=T=\left\{t_{0}, t_{1}, \ldots, t_{m-1}\right\}_{<}$, and then, for each $i<m$, let $T_{i}=T \backslash\left\{t_{i}\right\}$. Let $S_{i}$ be the intersection of all $T_{i}$-definable spheres $S$ such that $a \in S$. Since $a$ is not $T_{i}$-definable, there is $d$ such that $1 \leq d \leq n$ and $S_{i}$ is a $T_{i}$-definable $d$-sphere. We refer to $S_{i}$ as the $i$ th definable sphere of $a$. Notice that it is possible that $d=n$.

Clearly (or by the Hilbert Basis Theorem), the $i$ th definable sphere is $T_{i}$-definable. Hence, it is $T$-definable. There is a uniform way to define it, which we now describe. Fix some acceptable color $f: B \longrightarrow \mathbb{R}^{n}$, where $B \subseteq \mathbb{R}^{m}$, and also fix some $i<m$. Let $\theta_{f, i}(u, x, v)$ be an $(m+n+1)$-ary formula which asserts: $u \in B, v>0$ and $x$ is in the smallest sphere containing $\left\{f\left(u_{0}, u_{1}, \ldots, u_{i-1}, u, u_{i+1}, \ldots, u_{m-1}\right):\left\|u-u_{i}\right\|<v\right\}$. Then let $\varphi_{f, i}(u, x)$ be an $(m+n)$-ary formula asserting that $u \in B$ and $x$ is such that, for every $v>0, x$ is in the sphere defined by $\theta_{f, i}(u, x, v)$.

It is clear that if $t \in B$, then $\varphi_{f, i}(t, x)$ defines a sphere. Furthermore, if $a \in \mathbb{R}^{n}, f \in \mathcal{C}$ is an acceptable color for $a$, and $\operatorname{supp}(a)=\left\{t_{0}, t_{1}, \ldots, t_{m-1}\right\}$, then $\varphi_{f, i}(t, x)$ defines the $i$ th definable sphere of $a$. To see this last statement, let $S_{i}$ be the $i$ th definable sphere of $a$, and let $S_{i}^{\prime}$ be the sphere defined by $\varphi_{f, i}(t, x)$. Clearly, $S_{i} \subseteq S_{i}^{\prime}$. Now let $F: B \longrightarrow \mathbb{R}$ be the function where $F(u)=\inf \left\{\|f(u)-x\|^{2}: x \in S_{i}\right\}$. Then $F$ is a $T_{i}$-definable analytic function and $F(t)=0$. It then follows from Corollary 1.2 that $F\left(t^{\prime}\right)=0$ whenever $t^{\prime}$ agrees with $t$ except that perhaps $t_{i}^{\prime} \neq t_{i}$. This implies that $S_{i}^{\prime} \subseteq S_{i}$. Therefore, we have proved that $S_{i}=S_{i}^{\prime}$.

Suppose that $f: B \longrightarrow \mathbb{R}^{n}$ is an acceptable color, where $B \subseteq \mathbb{R}^{m}$. Making use of the formulas $\varphi_{f, i}(u, x)$, we define the functions $d_{f, i}: B \longrightarrow \omega$, and $r_{f, i}: B \longrightarrow \mathbb{R}$ such that whenever $t \in B$ and $S_{i}$ is the sphere defined by $\varphi_{f, i}(t, x)$, then $S_{i}$ is a $d_{f, i}(t)$ sphere of radius $r_{f, i}(t)$. All these functions are definable. We say that $f$ is preferred if, for each $i<m$, the functions $d_{f, i}$ and $r_{f, i}$ are analytic and whenever $u, v \in B$, then $r_{f, i}(u) \leq 2 r_{f, i}(v)$. Of course, if $d_{f, i}$ is analytic, then it is constant. A coloring $\chi: \mathbb{R}^{n} \longrightarrow \mathcal{C}$ is preferred if $\chi(x)$ is preferred for each $x \in \mathbb{R}^{n}$. Preferred colorings exist by Proposition 1.3. 
We make a further refinement of this definition. If $\varepsilon>0$ and $f: B \longrightarrow \mathbb{R}^{n}$ is a preferred color, where $B \subseteq \mathbb{R}^{m}$, then $f$ is $\varepsilon$-preferred if whenever $u, v \in B$ and $i<m$, then

$$
\|f(u)-f(v)\|<\varepsilon r_{f, i}(v) .
$$

The coloring $\chi$ is $\varepsilon$-preferred if $\chi(x)$ is $\varepsilon$-preferred for each $x \in \mathbb{R}^{n}$. Clearly, $\varepsilon$-preferred colorings exist.

The following is the main result of this section.

Theorem 3.1. Suppose $n \geq 3$, and suppose $Y=\left\{y_{0}, y_{1}, \ldots, y_{n}\right\} \subseteq \mathbb{F}^{n}$ is an $n$ simplex. There is $\varepsilon>0$ such that whenever $\mu: \mathbb{R}^{n} \longrightarrow \mathcal{C}$ is $\varepsilon$-preferred and $f_{0}, f_{1}, \ldots$, $f_{n-2} \in \mathcal{C}$, then there is a thin $\mathcal{C}_{2} \subseteq \mathcal{C}$ such that whenever $g \in \mathcal{C}$ and similarity $\alpha: \mathbb{R}^{n} \longrightarrow$ $\mathbb{R}^{n}$ are such that $f_{0}, f_{1}, \ldots, f_{n-2}$ are acceptable colors for $\alpha\left(y_{0}\right), \alpha\left(y_{1}\right), \ldots, \alpha\left(y_{n-2}\right)$, respectively, and $\mu\left(\alpha\left(y_{n-1}\right)\right)=\mu\left(\alpha\left(y_{n}\right)\right)=g$, then $g \in \mathcal{C}_{2}$.

Proof. Let $C_{n-1}$ be the set of $x \in \mathbb{R}^{n}$ such that $\left(y_{0}, y_{1}, \ldots, y_{n-2}, y_{n-1}\right) \mapsto\left(y_{0}, y_{1}, \ldots\right.$, $\left.y_{n-2}, x\right)$ is a similarity, and let $C_{n}$ be the set of $x \in \mathbb{R}^{n}$ such that $\left(y_{0}, y_{1}, \ldots, y_{n-2}, y_{n}\right) \mapsto$ $\left(y_{0}, y_{1}, \ldots, y_{n-2}, x\right)$ is a similarity. Each of $C_{n-1}$ and $C_{n}$ is a sphere with axis $H=$ $\operatorname{aff}\left(\left\{y_{0}, y_{1}, \ldots, y_{n-2}\right\}\right)$. Since $Y$ is an $n$-simplex, $\operatorname{dim}(H)=n-2$, so both $C_{n-1}, C_{n}$ are circles. Let $r_{n-1}, r_{n}$ be their respective radii. Let

$$
\varepsilon=\left\|y_{n}-y_{n-1}\right\| / 2 \max \left(r_{n}, r_{n-1}\right) .
$$

We will prove that this choice of $\varepsilon$ works.

For the remainder of this proof, fix an $\varepsilon$-preferred coloring $\mu$ and colors $f_{0}, f_{1}, \ldots$, $f_{n-2} \in \mathcal{C}$. Let $\operatorname{dom}\left(f_{i}\right)=B_{i}=I_{i 0} \times I_{i 1} \times \cdots \times I_{i m_{i}-1}$ for $i \leq n-2$. We say that $\left(a_{0}, a_{1}, \ldots, a_{n}\right) \in\left(\mathbb{R}^{n}\right)^{n+1}$ is nice if $\left(y_{0}, y_{1}, \ldots, y_{n}\right) \mapsto\left(a_{0}, a_{1}, \ldots, a_{n}\right)$ is a similarity, $f_{0}, f_{1}, \ldots, f_{n-2}$ are acceptable colors for $a_{0}, a_{1}, \ldots, a_{n-2}$, respectively, and $\mu\left(a_{n-1}\right)=$ $\mu\left(a_{n}\right)$.

Lemma 3.1.1. If $\left(a_{0}, a_{1}, \ldots, a_{n}\right)$ is nice, then $\operatorname{supp}\left(a_{n-1}\right) \cup \operatorname{supp}\left(a_{n}\right) \subseteq \operatorname{supp}\left(a_{0}\right) \cup$ $\operatorname{supp}\left(a_{1}\right) \cup \cdots \cup \operatorname{supp}\left(a_{n-2}\right)$.

Proof. It suffices to prove that $\operatorname{supp}\left(a_{n}\right) \subseteq \operatorname{supp}\left(a_{0}\right) \cup \operatorname{supp}\left(a_{1}\right) \cup \cdots \cup \operatorname{supp}\left(a_{n-2}\right)$, for then by a similar proof (or by symmetry), we can also conclude that $\operatorname{supp}\left(a_{n-1}\right) \subseteq$ $\operatorname{supp}\left(a_{0}\right) \cup \operatorname{supp}\left(a_{1}\right) \cup \cdots \cup \operatorname{supp}\left(a_{n-2}\right)$.

Let $\alpha: \mathbb{R}^{n} \longrightarrow \mathbb{R}^{n}$ be a similarity such that $\alpha\left(y_{i}\right)=a_{i}$ for $i \leq n$, and let $c$ be its scaling factor. For each $i \leq n$, let $\operatorname{supp}\left(a_{i}\right)=\left\{t_{i 0}, t_{i 1}, \ldots, t_{i m_{i}-1}\right\}_{<}$. For a contradiction, suppose $j<m_{n}$ is such that $t_{n j} \notin \operatorname{supp}\left(a_{i}\right)$ for every $i \leq n-2$. Let $g=\mu\left(a_{n-1}\right)=\mu\left(a_{n}\right)$, where $g: B \longrightarrow \mathbb{R}^{n}$. For $1 \leq i \leq n-2$, define $F_{i}: B_{0} \times B_{i} \times B \longrightarrow \mathbb{R}$ by

$$
F_{i}(u, v, w)=\left\|y_{0}-y_{i}\right\|^{2}\left\|f_{0}(u)-g(w)\right\|^{2}-\left\|y_{0}-y_{n}\right\|^{2}\left\|f_{0}(u)-f_{i}(v)\right\|^{2},
$$

so that $F_{i}$ is a definable analytic function. Clearly, $F_{i}\left(t_{0}, t_{i}, t_{n}\right)=0$. Thus, by Corollary 1.2, if we let $t_{n}^{\prime}$ be obtained from $t_{n}$ by keeping all coordinates fixed except that $t_{n j}$ is replaced by a number sufficiently close to it, then we get that $F_{i}\left(t_{0}, t_{i}, t_{n}^{\prime}\right)=0$. It follows from this, by Corollary 1.2 and Proposition 1.3, that the $j$ th definable sphere of $a_{n}$ is the 
circle $C=\alpha\left(C_{n}\right)$ having radius $r=c r_{n}$. Since $\mu$ is preferred, the $j$ th definable sphere $C^{\prime}$ of $a_{n-1}$ is also a circle. Let $r^{\prime}$ be the radius of $C^{\prime}$, and, since $\mu$ is preferred, $r^{\prime} \leq 2 r$. Since $\mu$ is $\varepsilon$-preferred, we get that

$$
\begin{aligned}
\varepsilon & >\left\|a_{n}-a_{n-1}\right\| / \max \left(r, r^{\prime}\right) \\
& \geq\left\|a_{n}-a_{n-1}\right\| / 2 r \\
& =\left\|y_{n}-y_{n-1}\right\| / 2 r_{n} \geq \varepsilon,
\end{aligned}
$$

which is a contradiction.

Lemma 3.1.2. If $a_{0}, a_{1}, \ldots, a_{n-2} \in \mathbb{R}^{n}$, then there are only finitely many pairs $\left(a_{n-1}, a_{n}\right)$ such that $\left(a_{0}, a_{1} \ldots, a_{n}\right)$ is nice.

Proof. We can assume that there is at least one pair $\left(a_{n-1}, a_{n}\right)$ such that $\left(a_{0}, a_{1}, \ldots, a_{n}\right)$ is nice. Hence, we can let $\alpha: \mathbb{R}^{n} \longrightarrow \mathbb{R}^{n}$ be a similarity such that $\alpha\left(y_{i}\right)=a_{i}$ for each $i \leq n-2$. Then $c=\left\|a_{0}-a_{1}\right\| /\left\|y_{0}-y_{1}\right\|$ is its scaling factor.

Let $T=\operatorname{supp}\left(a_{0}\right) \cup \operatorname{supp}\left(a_{1}\right) \cup \cdots \cup \operatorname{supp}\left(a_{n-2}\right)$, which is finite. Suppose, for a contradiction, that there are infinitely many pairs $\left(a_{n-1}, a_{n}\right)$ such that $\left(a_{0}, a_{1} \ldots, a_{n}\right)$ is nice.

By Lemma 3.1.1, for each such pair, $\operatorname{supp}\left(a_{n-1}\right) \cup \operatorname{supp}\left(a_{n}\right) \subseteq T$ and, moreover, $\left|\operatorname{supp}\left(a_{n-1}\right)\right|=\left|\operatorname{supp}\left(a_{n}\right)\right|$ and $\operatorname{supp}\left(a_{n-1}\right) \neq \operatorname{supp}\left(a_{n}\right)$. Thus, there are $m<k$,

$$
\begin{aligned}
T_{n} & =\left\{t_{0}, t_{1}, \ldots, t_{m-1}\right\}_{<} \subseteq T, \\
T_{n-1} & =\left\{t_{0}^{\prime}, t_{1}^{\prime}, \ldots, t_{m-1}^{\prime}\right\}_{<} \subseteq T,
\end{aligned}
$$

and three distinct such pairs $\left(a_{n-1}, a_{n}\right),\left(a_{n-1}^{\prime}, a_{n}^{\prime}\right),\left(a_{n-1}^{\prime \prime}, a_{n}^{\prime \prime}\right)$ such that $\operatorname{supp}\left(a_{n}\right)=$ $\operatorname{supp}\left(a_{n}^{\prime}\right)=\operatorname{supp}\left(a_{n}^{\prime \prime}\right)=T_{n}$ and $\operatorname{supp}\left(a_{n-1}\right)=\operatorname{supp}\left(a_{n-1}^{\prime}\right)=\operatorname{supp}\left(a_{n-1}^{\prime \prime}\right)=T_{n-1}$. Notice that not only are these three pairs distinct, but also $a_{n} \neq a_{n}^{\prime} \neq a_{n}^{\prime \prime} \neq a_{n}$ and $a_{n-1} \neq a_{n-1}^{\prime} \neq a_{n-1}^{\prime \prime} \neq a_{n-1}$.

Clearly, $T_{n} \neq T_{n-1}$ and $\left|T_{n}\right|=\left|T_{n-1}\right|$, so $m \geq 1$. Let $g=\mu\left(a_{n}\right)=\mu\left(a_{n-1}\right)$, $g^{\prime}=\mu\left(a_{n}^{\prime}\right)=\mu\left(a_{n-1}^{\prime}\right)$, and $g^{\prime \prime}=\mu\left(a_{n}^{\prime \prime}\right)=\mu\left(a_{n-1}^{\prime \prime}\right)$. Let $B=\operatorname{dom}(g) \cap \operatorname{dom}\left(g^{\prime}\right) \cap$ $\operatorname{dom}\left(g^{\prime \prime}\right) \subseteq \mathbb{R}^{m}$. Clearly, $t, t^{\prime} \in B$.

Let $C$ be the unique circle for which $a_{n}, a_{n}^{\prime}, a_{n}^{\prime \prime} \in C$, and let $C^{\prime}$ be the unique circle for which $a_{n-1}, a_{n-1}^{\prime}, a_{n-1}^{\prime \prime} \in C^{\prime}$. Clearly, $C=\alpha\left(C_{n}\right)$ and $C^{\prime}=\alpha\left(C_{n-1}\right)$. The radii of $C$ and $C^{\prime}$ are $c r_{n}$ and $c r_{n-1}$ respectively, and both of these circles have the same axis, namely $\alpha(H)=\operatorname{aff}\left(\left\{a_{0}, a_{1}, \ldots, a_{n-2}\right\}\right)$. Since $c r_{n}$ is the radius of $C$, it is $T_{n}$-definable, so that $c$ is $T_{n}$-definable. Similarly, $c$ is $T_{n-1}$-definable, so $c$ is $\left(T_{n} \cap T_{n-1}\right)$-definable.

Let $T_{n} \backslash T_{n-1}=\left\{s_{0}, s_{1}, \ldots, s_{k}\right\}_{<}$, and let $T_{n-1} \backslash T_{n}=\left\{s_{0}^{\prime}, s_{1}^{\prime}, \ldots, s_{k}^{\prime}\right\}_{<}$. Let $J_{0}, J_{1}, \ldots$, $J_{k}$ be those factors of $B$ such that $s, s^{\prime} \in J_{0} \times J_{1} \times \cdots \times J_{k}$. Let $B^{\prime}=J_{0} \times J_{1} \times \cdots \times$ $J_{k}$. Let $h: B^{\prime} \longrightarrow \mathbb{R}^{n}$ be the $\left(T_{n} \cap T_{n-1}\right)$-definable function which is a "slice" of $g: B \longrightarrow \mathbb{R}^{n}$ defined as follows: if $\left(u_{0}, u_{1}, \ldots, u_{k}\right) \in B^{\prime}$, then $h\left(u_{0}, u_{1}, \ldots, u_{k}\right)=$ $g\left(x_{0}, x_{1}, \ldots, x_{m-1}\right)$, where $x_{i}=t_{i}$ if $t_{i} \in T_{n} \cap T_{n-1}$ and $x_{i}=u_{j}$ if $x_{i} \in J_{j}$.

Now let $F:\left(B^{\prime}\right)^{2} \longrightarrow \mathbb{R}$ be such that

$$
F(u, v)=\|h(u)-h(v)\|^{2}-c^{2}\left\|y_{n}-y_{n-1}\right\|^{2} .
$$


Then $F$ is a $\left(T_{n} \cap T_{n-1}\right)$-definable analytic function, and $F\left(s, s^{\prime}\right)=0$. By Corollary 1.2, $F$ is constantly 0 on $\left(B^{\prime}\right)^{2}$. In particular, $0=F(s, s)=-c^{2}\left\|y_{n}-y_{n-1}\right\|^{2}$ implying that $y_{n}=y_{n-1}$, which is a contradiction.

We wish to define the profile of a nice $\left(a_{0}, a_{1}, \ldots, a_{n}\right)$. Recall that the boxes $B_{i}$ and intervals $I_{i j}$, where $i \leq n-2$ and $j<m_{i}$, have already been defined (in the second paragraph of the proof of Theorem 3.1). Let $B_{n}=\operatorname{dom}\left(\mu\left(a_{n}\right)\right) \subseteq \mathbb{R}^{m_{n}}$. Then also $B_{n}=\operatorname{dom}\left(\mu\left(a_{n-1}\right)\right)$, so let $m_{n-1}=m_{n}$. Then let $\operatorname{supp}\left(a_{i}\right)=\left\{t_{i 0}, t_{i 1}, \ldots, t_{i m_{i}-1}\right\}_{<}$. Let $P=\left\{\langle i, j\rangle: i \leq n\right.$ and $\left.j<m_{i}\right\}$, and let $E$ be the equivalence relation on $P$, where $\langle i, j\rangle,\left\langle i^{\prime}, j^{\prime}\right\rangle$ are equivalent iff $t_{i j}=t_{i^{\prime} j^{\prime}}$. Then the profile of $\left(a_{0}, a_{1}, \ldots, a_{n}\right)$ is the pair $\left\langle E, B_{n}\right\rangle$.

Clearly, for any box $B$, there are at most finitely many possible profiles $\langle E, B\rangle$.

Lemma 3.1.3. Suppose $\left(a_{0}, a_{1}, \ldots, a_{n}\right),\left(b_{0}, b_{1}, \ldots, b_{n}\right)$ are nice and have the same profiles. Then there are $a_{n-1}^{\prime}, a_{n}^{\prime}$ such that $\left(a_{0}, a_{1}, \ldots, a_{n-2}, a_{n-1}^{\prime}, a_{n}^{\prime}\right)$ is nice and $\mu\left(a_{n}^{\prime}\right)=\mu\left(b_{n}\right)$.

Proof. Let $g=\mu\left(a_{n-1}\right)=\mu\left(a_{n}\right)$ and $h=\mu\left(b_{n-1}\right)=\mu\left(b_{n}\right)$, and then let $B=$ $\operatorname{dom}(g)=\operatorname{dom}(h)$. For $i \leq n$, let

$$
\begin{aligned}
& \operatorname{supp}\left(a_{i}\right)=\left\{t_{i 0}, t_{i 1}, \ldots, t_{i m_{i}-1}\right\}_{<}, \\
& \operatorname{supp}\left(b_{i}\right)=\left\{u_{i 0}, u_{i 1}, \ldots, u_{i m_{i}-1}\right\}_{<} .
\end{aligned}
$$

For $i \leq n-2$ and $e \in\{n-1, n\}$, let $F_{e i}: B_{0} \times B_{1} \times \cdots \times B_{n-2} \times B^{2} \longrightarrow \mathbb{R}$ be such that if $x=\left(x_{0}, x_{1}, \ldots, x_{n}\right) \in B_{0} \times B_{1} \times \cdots \times B_{n-2} \times B^{2}$, then

$$
F_{e i}(x)=\left\|y_{0}-y_{1}\right\|^{2}\left\|h\left(x_{e}\right)-f_{i}\left(x_{i}\right)\right\|^{2}-\left\|y_{e}-y_{i}\right\|^{2}\left\|f_{0}\left(x_{0}\right)-f_{1}\left(x_{1}\right)\right\|^{2} .
$$

Also, define $F: B_{0} \times B_{1} \times \cdots \times B_{n-2} \times B^{2} \longrightarrow \mathbb{R}$ by

$$
F(x)=\left\|y_{0}-y_{1}\right\|^{2}\left\|h\left(x_{n-1}\right)-h\left(x_{n}\right)\right\|^{2}-\left\|y_{n-1}-y_{n}\right\|^{2}\left\|f_{0}\left(x_{0}\right)-f_{1}\left(x_{1}\right)\right\|^{2} .
$$

Then $F$ and each $F_{e i}$ are definable analytic functions and

$$
F\left(u_{0}, u_{1}, \ldots, u_{n}\right)=F_{e i}\left(u_{0}, u_{1}, \ldots, u_{n}\right)=0 .
$$

Since $\left(a_{0}, a_{1}, \ldots, a_{n}\right),\left(b_{0}, b_{1}, \ldots, b_{n}\right)$ have the same profiles, Corollary 1.2 can be invoked to show that

$$
F\left(t_{0}, t_{1}, \ldots, t_{n}\right)=F_{e i}\left(t_{0}, t_{1}, \ldots, t_{n}\right)=0 .
$$

Letting $a_{n-1}^{\prime}=h\left(t_{n-1}\right)$ and $a_{n}^{\prime}=h\left(t_{n}\right)$, we then easily see that $\left(a_{0}, a_{1}, \ldots, a_{n-2}\right.$, $\left.a_{n-1}^{\prime}, a_{n}^{\prime}\right)$ is nice and $\mu\left(a_{n}^{\prime}\right)=h$.

We now complete the proof of Theorem 3.1. Let $\mathcal{C}_{2}$ be the set of $g \in \mathcal{C}$ for which there is a nice $\left(a_{0}, a_{1}, \ldots, a_{n}\right)$ such that $g=\mu\left(a_{n}\right)$. If $\mathcal{C}_{2}$ is not thin, then let $k<\omega$ and $g_{0}, g_{1}, g_{2}, \ldots$ be infinitely many distinct colors in $\mathcal{C}_{2}$ such that $v\left(g_{i}\right)=k$ for every $i<\omega$. 
For each $i<\omega$, let $\left(a_{i 0}, a_{i 1}, \ldots, a_{i n}\right)$ be nice such that $\mu\left(a_{i n}\right)=g_{i}$. It follows from Lemma 3.1.1 that there are only finitely many boxes such that each $\operatorname{dom}\left(g_{i}\right)$ is one of them. Thus, without loss, we can assume that there is a single $B$ such that $\operatorname{dom}\left(g_{i}\right)=B$ for each $i<\omega$. Since there are only finitely many profiles $\langle E, B\rangle$, we can further assume that each $\left(a_{i 0}, a_{i 1}, \ldots, a_{i n}\right)$ has the same profile. Then, by Lemma 3.1.3, for each $i<\omega$, there are $a_{i n-1}^{\prime}, a_{i n}^{\prime}$ such that $\left(a_{00}, a_{01}, \ldots, a_{0 n-2}, a_{i n-1}^{\prime}, a_{i n}^{\prime}\right)$ is nice and $\mu\left(a_{i n}^{\prime}\right)=g_{i}$. Since the $g_{i}$ 's are distinct, so must the $a_{i n}^{\prime}$ 's be distinct. However, this contradicts Lemma 3.1.2, thereby proving the theorem.

\section{Proof of Theorem D}

This section contains the proof of Theorem D. Since, as previously mentioned, Komjáth [5] has already proved Theorem $\mathrm{D}$ in case $n=2$, we assume throughout this section that $n \geq 3$.

To begin the proof of Theorem D, let $X, Y \subseteq \mathbb{R}^{n}$ be as in that theorem; that is, $X$ is infinite and $Y$ is an $n$-simplex. By taking a subset of $X$ if needed, assume that $X$ is countable, and then let $\mathbb{F} \subseteq \mathbb{R}$ be a countable, real-closed subfield such that $X, Y \subseteq \mathbb{F}^{n}$, and then the assumptions from the previous sections can be made.

Lemma 4.1. Let $\chi: \mathbb{R}^{n} \longrightarrow \mathcal{C}$ be an acceptable coloring. If $\alpha: \mathbb{R}^{n} \longrightarrow \mathbb{R}^{n}$ is a similarity and $f \in \mathcal{C}$, then $\{a \in X: \chi(\alpha(a))=f\}$ is finite.

Proof. Let $f$ be $m$-ary, and let $k=|\operatorname{supp}(\alpha(Y))|$. If $a \in X$, then by Lemma 2.3, $\operatorname{supp}(\alpha(a)) \subseteq \operatorname{supp}(Y)$, so that $m \leq k$. Therefore, it follows that $\mid\{a \in X: \chi(\alpha(a))=$ $f\} \mid \leq\left(\begin{array}{c}k \\ m\end{array}\right)$.

For the rest of this section, we fix $\varepsilon>0$ that will work in Theorem 3.1 no matter how we write $Y$ as $\left\{y_{0}, y_{1}, \ldots, y_{n}\right\}$. However, now fix $Y=\left\{y_{0}, y_{1}, \ldots, y_{n}\right\}$. We let $\mu$ be the $\varepsilon$-preferred coloring that is minimal in the following sense: whenever $\mu^{\prime}$ is an $\varepsilon$-preferred coloring and $a \in \mathbb{R}^{n}$, then $v(\mu(a)) \leq v\left(\mu^{\prime}(a)\right)$.

Of course, there are minimal $\varepsilon$-preferred colorings of any $\mathbb{R}^{k}$, where $1 \leq k<\omega$, not just for $\mathbb{R}^{n}$. If $1 \leq k, m<\omega$, then there is a canonical bijection from $\mathbb{R}^{k m}$ to $\left(\mathbb{R}^{k}\right)^{m}$, giving us a canonical way to extend $\mu: \mathbb{R}^{k} \longrightarrow \mathcal{C}_{k}$ to $\mu:\left(\mathbb{R}^{k}\right)^{m} \longrightarrow \mathcal{C}_{k m}$.

We refer to elements of $\mathcal{C}_{n(n+1)}$ as types. We say that the similarity $\alpha: \mathbb{R}^{n} \longrightarrow \mathbb{R}^{n}$ has type $\tau$ (in symbols: type $(\alpha)=\tau$ ) if $\tau$ is a type and $\mu\left(\alpha\left(y_{0}\right), \alpha\left(y_{1}\right), \ldots, \alpha\left(y_{n}\right)\right)=\tau$.

Lemma 4.2. If $a \in X$ and type $(\alpha)=$ type $(\beta)$, then $\mu(\alpha(a))=\mu(\beta(a))$.

Proof. Suppose the lemma is false; thus, type $(\alpha)=$ type $(\beta)=\tau$ and $\mu(\alpha(a)) \neq$ $\mu(\beta(a))$. By Lemma 2.3, let $F:\left(\mathbb{R}^{n}\right)^{n+1} \longrightarrow \mathbb{R}^{n}$ be a definable analytic function such that $a=F\left(y_{0}, y_{1}, \ldots, y_{n}\right), \alpha(a)=F\left(\alpha\left(y_{0}\right), \alpha\left(y_{1}\right), \ldots, \alpha\left(y_{n}\right)\right)$ and $\beta(a)=$ $F\left(\beta\left(y_{0}\right), \beta\left(y_{1}\right), \ldots, \beta\left(y_{n}\right)\right)$.

Let $\operatorname{dom}(\tau)=B=I_{0} \times I_{1} \times \cdots \times I_{m-1} \subseteq \mathbb{R}^{m}$, and let

$$
\operatorname{supp}\left(\alpha\left(y_{0}\right), \alpha\left(y_{1}\right), \ldots, \alpha\left(y_{n}\right)\right)=\left\{s_{0}, s_{1}, \ldots, s_{m-1}\right\}_{<}
$$


and

$$
\operatorname{supp}\left(\beta\left(y_{0}\right), \beta\left(y_{1}\right), \ldots, \beta\left(y_{n}\right)\right)=\left\{t_{0}, t_{1}, \ldots, t_{m-1}\right\}_{<} .
$$

Let $\tau=\left(\tau_{0}, \tau_{1}, \ldots, \tau_{n}\right)$, where each $\tau_{i}: B \longrightarrow \mathbb{R}^{n}$, and then let $G: B \longrightarrow \mathbb{R}^{n}$ be the definable analytic function such that

$$
G\left(x_{0}, x_{1}, \ldots, x_{m-1}\right)=F\left(\tau_{0}(x), \tau_{1}(x), \ldots, \tau_{n}(x)\right) .
$$

In particular, $\alpha(a)=G\left(s_{0}, s_{1}, \ldots, s_{m-1}\right)$ and $\beta(a)=G\left(t_{0}, t_{1}, \ldots, t_{m-1}\right)$.

Let $J=\left\{i<m: s_{i} \in \operatorname{supp}(\alpha(a))\right\}$ and $K=\left\{i<m: t_{i} \in \operatorname{supp}(\beta(a))\right\}$. We will show that $J=K$.

Define $s^{\prime}, t^{\prime} \in B$ as follows: let $s_{i}^{\prime}=s_{i}$ and $t_{i}^{\prime}=t_{i}$ if $i \in J$, and let $s_{i}^{\prime}, t_{i}^{\prime}$ be the midpoint of $I_{i}$ if $i \notin J$. Then $G\left(s_{0}^{\prime}, s_{1}^{\prime}, \ldots, s_{m-1}^{\prime}\right)=G\left(s_{0}, s_{1}, \ldots, s_{m-1}\right)$, so by Corollary 1.2, $G\left(t_{0}^{\prime}, t_{1}^{\prime}, \ldots, t_{m-1}^{\prime}\right)=G\left(t_{0}, t_{1}, \ldots, t_{m-1}\right)$. Thus, $K \subseteq J$. Then, by symmetry, $J=K$.

Let $J=\left\{j_{0}, j_{1}, \ldots, j_{k-1}\right\}_{<}$, and let $B^{\prime}=I_{j_{0}} \times I_{j_{1}} \times \cdots \times I_{j_{k-1}} \subseteq \mathbb{R}^{k}$, and let $g: B^{\prime} \longrightarrow$ $\mathbb{R}^{n}$ be such that $g\left(x_{0}, x_{1}, \ldots, x_{k-1}\right)=G\left(y_{0}, y_{1}, \ldots, y_{m-1}\right)$, where $y_{j}=x_{i}$ if $j=j_{i}$ and $y_{j}=s_{j}^{\prime}$ if $j \notin J$. Then, $g$ is a definable analytic function and $\alpha(a)=g\left(s_{0}, s_{1}, \ldots, s_{m-1}\right)$ and $\beta(a)=g\left(t_{0}, t_{1}, \ldots, t_{m-1}\right)$. Thus, $g \subseteq \mu(\alpha(a))$ and $g \subseteq \mu(\beta(a))$. Since $\mu$ is a preferred coloring, then $\mu(\alpha(a))=\mu(\beta(a))$.

We say that a function $\rho: D \longrightarrow 2$ is good if $D \subseteq \mathcal{C}$ is finite and whenever $\alpha: Y \longrightarrow \mathbb{R}^{n}$ is a similarity and $\mu(\alpha(Y)) \subseteq D$, then $\rho(\mu(\alpha(y)))=0$ for some $y \in Y$.

Lemma 4.3. Suppose that $\rho$ is good and that $\tau$ is a type. Then there is a good $\rho^{\prime} \supseteq \rho$ and $a \in X$ such that whenever $\alpha: \mathbb{R}^{n} \longrightarrow \mathbb{R}^{n}$ is a similarity and type $(\alpha)=\tau$, then $\rho^{\prime}(\mu(\alpha(a)))=1$.

Proof. Let $D_{0}=\operatorname{dom}(\rho)$, which is finite since $\rho$ is good.

By Corollary 2.6, let $D_{1} \subseteq \mathcal{C}$ be a thin set such that whenever $j \leq n$ and $\alpha: \mathbb{R}^{n} \longrightarrow \mathbb{R}^{n}$ is a similarity for which $\mu\left(\alpha\left(y_{i}\right)\right) \in D_{0}$ if $j \neq i \leq n$, then $\mu\left(\alpha\left(y_{j}\right)\right) \in D_{1}$.

By Theorem 3.1, let $D_{2} \subseteq \mathcal{C}$ be a thin set such that whenever $j<k \leq n$ and $\alpha: \mathbb{R}^{n} \longrightarrow \mathbb{R}^{n}$ is a similarity for which $\mu\left(\alpha\left(y_{i}\right)\right) \in D_{0}$ if $i \leq n$ and $i \notin\{j, k\}$, and $\mu\left(\alpha\left(y_{j}\right)\right)=\mu\left(\alpha\left(y_{k}\right)\right)$, then $\mu\left(\alpha\left(y_{j}\right)\right) \in D_{2}$.

It follows from Lemmas 4.1 and 4.2 that we can let $a \in X$ and $f \notin D_{0} \cup D_{1} \cup D_{2}$ be such that $\mu(\alpha(a))=f$ whenever type $(\alpha)=\tau$. Now let $\rho^{\prime} \supseteq \rho$ be such that $\operatorname{dom}\left(\rho^{\prime}\right)=D_{0} \cup\{f\}$ and $\rho^{\prime}(f)=1$.

We claim that $\rho^{\prime}$ is good. If it were not, then there would be a similarity $\alpha$ such that $\alpha(Y) \subseteq \operatorname{dom}\left(\rho^{\prime}\right)$ and $\rho^{\prime}(\alpha(y))=1$ for each $y \in Y$. Let $k=|\{y \in Y: \mu(y)=f\}|$. We consider several cases.

$k=0$. Then $\rho(\alpha(y))=1$ for all $y \in Y$, contradicting that $\rho$ is good.

$k=1$. Then $f \in D_{1}$, which is a contradiction.

$k=2$. Then $f \in D_{2}$, which is a contradiction.

$k \geq 3$. This contradicts Lemma 2.1 .

Thus, $\rho^{\prime}$ is good.

That $\rho^{\prime}$ has the required property is clear since whenever type $(\alpha)=\tau$, then $\rho^{\prime}(\mu(\alpha(a)))=\rho^{\prime}(f)=1$. 
Our goal now is to construct a function $\rho: \mathcal{C} \longrightarrow 2$ and then let $\psi=\rho \circ \mu$. If this is done properly, then $\psi$ will be a 2-coloring of $\mathbb{R}^{n}$ that confirms Theorem $\mathrm{D}$. The function $\rho$ will be constructed as the union of an increasing sequence $\rho_{0} \subseteq \rho_{1} \subseteq \cdots$ of good functions. The construction will proceed by stages: at stage $i$, where $i<\omega$, we will construct $\rho_{i}$.

We need a list $\left\langle f_{j}: j<\omega\right\rangle$ of all the colors in $\mathcal{C}$, and we also need a list $\left\langle\tau_{j}: j<\omega\right\rangle$ of all types.

To start this construction, we let $\rho_{0}$ be the empty function, which is good vacuously. The construction now splits into even and odd stages.

The odd stages. Suppose $i=2 j$. Then let $\rho_{i+1} \supseteq \rho_{i}$ be such that $\operatorname{dom}\left(\rho_{i+1}\right)=$ $\operatorname{dom}\left(\rho_{i}\right) \cup\left\{f_{j}\right\}$ and $\rho_{i+1}\left(f_{j}\right)=0$. It is easily checked that $\rho_{i+1}$ is good.

The even stages. Suppose $i=2 j+1$. Use Lemma 4.3 to get a good $\rho_{i+1} \supseteq \rho_{i}$. That is, whenever $\alpha: \mathbb{R}^{n} \longrightarrow \mathbb{R}^{n}$ is such that type $(\alpha)=\tau_{j}$, then there is $a \in X$ such that $\mu(\alpha(a)) \in \operatorname{dom}\left(\rho_{i+1}\right)$ and $\rho_{i+1}(\mu(\alpha(a)))=1$.

We have completed the construction of the sequence $\left\langle\rho_{i}: i<\omega\right\rangle$. Let $\rho$ be its union, and let $\psi=\rho \circ \mu$.

The odd stages assure that $\operatorname{dom}(\rho)=\mathcal{C}$ and, therefore, that $\operatorname{dom}(\psi)=\mathbb{R}^{n}$. We now show that the function $\psi: \mathbb{R}^{n} \longrightarrow 2$ confirms Theorem D. Let $\alpha: \mathbb{R}^{n} \longrightarrow \mathbb{R}^{n}$ be a similarity, and suppose type $(\alpha)=\tau_{j}$. Let $X^{\prime}=\alpha(X)$ and $Y^{\prime}=\alpha(Y)$. We wish to show that there are $a^{\prime} \in X^{\prime}$ and $y^{\prime} \in Y^{\prime}$ such that $\psi\left(a^{\prime}\right)=1$ and $\psi\left(y^{\prime}\right)=0$.

Since $Y^{\prime}$ is finite, the odd stages assure that there is $i<\omega$ such that $i \geq 2 j+1$ and $\mu\left(Y^{\prime}\right) \subseteq \operatorname{dom}\left(\rho_{i}\right)$. Since $\rho_{i}$ is good, there is $y^{\prime} \in Y^{\prime}$ such that $\rho_{i}\left(\mu\left(y^{\prime}\right)\right)=0$. Thus, $\psi\left(y^{\prime}\right)=0$. The even stages assure that there is $a^{\prime} \in X^{\prime}$ such that $\mu\left(a^{\prime}\right) \in \operatorname{dom}\left(\rho_{i}\right)$ and $\rho\left(\mu\left(a^{\prime}\right)\right)=1$. Thus, $\psi\left(a^{\prime}\right)=1$.

This completes the proof of Theorem D.

\section{References}

1. Baumgartner, J.E., Partitioning vector spaces, J. Combin. Theory Ser. A 18 (1975), 231-233.

2. Ceder, J., Finite subsets and countable decompositions of Euclidean space, Rev. Roumaine Math. Pures Appl. 14 (1969), 1247-1251.

3. van den Dries, L., Tame Topology and O-Minimal Structures, London Mathematical Society Lecture Note Series 248, Cambridge University Press, Cambridge, 1998.

4. Erdős, P., Graham, R.L., Montgomery, P., Rothschild, B.L., Spencer, J., Straus, E.G., Euclidean Ramsey Theorems. II, Infinite and Finite Sets (Keszthely, 1973), Vol. I, Colloq. Math. Soc. János Bolyai, Vol. 10, North-Holland, Amsterdam, 1975, pp. 529-557.

5. Komjáth, P., Personal communication, October, 2005.

6. Rado, R., Note on combinatorial analysis, Proc. London Math. Soc. (2) 48 (1943), 122-160.

7. Schmerl, J.H., Partitioning Euclidean space, Discrete Comput. Geom. 10 (1993), 101-106.

8. Schmerl, J.H., Countable partitions of Euclidean space. Math. Proc. Cambridge Philos. Soc. 120 (1996), 7-12.

9. Witt, E., Ein kombinatorischer Satz der Elementargeometrie, Math. Nachr. 6 (1952), 261-262. 